\title{
Incentives to (irreversible) investments under different regulatory regimes*
}

\author{
Paolo Panteghini and Carlo Scarpa \\ Dipartimento di Scienze Economiche \\ Università di Brescia \\ V. S. Faustino, $74 \mathrm{~b}$ \\ 25122 Brescia (Italy) \\ panteghi@eco.unibs.it \\ cscarpa@eco.unibs.it
}

January 9, 2002

\begin{abstract}
This paper addresses the issue of how regulatory constraints affect firm's investment choices when the firm has the option to delay investment. The $R P I-x$ rule is compared to a profit sharing rule, which increases the $x$ factor in case profits go beyond a given level. It is shown that these rules are identical in their impact on investment choices, in that the change in the option value exactly compensates the change in the "direct" profitability of investment. The result is then analysed in the light of option theory and explained on the basis of the "bad news principle".
\end{abstract}

JEL Classification: L51, D92, G31

Keywords: regulation, investment, RPI-x, profit sharing.

*We would like to thank Giacomo Calzolari, Vesa Kanniainen, Pia Koskenoja, Michele Moretto and participants to seminars at the Universities of Bologna, Helsinki and Padova and at CESifo in Munich for comments on earlier drafts. 


\section{Introduction}

The literature on investment incentives in regulated industries tends to indicate how a price cap such as the by now traditional $R P I-x$ provides the regulated firm appropriate incentives to invest. The idea is that the regulated price should increase at a rate equal to the difference between the expected inflation rate (the Retail Price Index, $R P I$ ) and an exogenously given component $(x)$ which, roughly speaking, represents the expected increase of productivity the firm should attain. By making prices insensitive at the margin to firm's choices, the $R P I-x$ rule appears to eliminate the downward bias and the phenomenon known as "underinvestment". As Beesley and Littlechild (1989) put it when listing the main arguments in favor of $R P I-x$, "Because the company has the right to keep whatever profits it can earn during the specified period (and must also absorb any losses), this preserves the incentive to productive efficiency associated with unconstrained profit maximization".

However, we know from the UK experience that an $R P I-x$ scheme tends to leave the firm large profits, so that some authors ${ }^{1}$ (among others, Sappington and Weisman, 1996; Burns et al., 1998) have brought into consideration an alternative called "sliding scale" (or, less cryptically, "profit sharing"). According to this scheme, in case the firm's profits go beyond a pre-specified level, the $x$ factor should be automatically adjusted upwards, making the price-cap more stringent; this re-distributes rents to the consumers, making the system more "fair" and more sustainable from a political viewpoint. This proposal has been criticized by some authors (see e.g., Mayer and Vickers, 1996) who - among other things - stress that if higher investment spurs a tighter price cap, then we have a dis-incentive to invest. The superiority of the $R P I-x$ system relative to profit sharing rules on the ground of technical efficiency was recognized also by advocates of profit sharing rules (e.g., Lyon, 1996), who only defend the PS system on the basis of overall allocative efficiency (profit sharing "typically" increases consumers surplus). Weisman (1993) shows that when price cap rules incorporate an element of profit sharing, price caps may represent a worsening relative to a pure cost based regulation (a notoriously inefficient set-up).

While most papers in the regulation literature implicitly consider fully reversible investments, we take a different approach which, following the modern theory of investment, stresses how these choices are typically irreversible $^{2}$. The consequence of irreversibility is that the decision to invest

\footnotetext{
${ }^{1}$ This proposal, already debated in the UK, has also become popular among several policy makers, such as the Italian electricity regulator.

${ }^{2}$ As stated by Dixit and Pindyck (1994, p.3) "Most investment decisions share three
} 
should consider the option value of investment. As an irreversible choice entails burning an opportunity, the value of waiting should be considered.

Our study shows that considering this aspect considerably changes the relative desirability of the aforementioned regulatory policies. With irreversible investments, we can show that the effect of $R P I-x$ and profit sharing on the incentives to invest may well be identical. The reason is that the introduction of the profit ceiling into a $R P I-x$ scheme decreases the net present value of the investment, but also decreases the value of waiting (i.e., the option value) by exactly the same amount. This is an application of the "bad news principle" (Bernanke, 1983), which indicates that, under investment irreversibility, uncertainty acts asymmetrically since only the unfavorable events affect the current propensity to invest. If, thus, profit sharing (i.e., the change in the $x$ factor) occurs only in the good state, investment decisions are not affected.

This paper is linked to two streams of literature. The first one is the literature on investment irreversibility. Irreversibility may arise from 'lemon effects' (second-hand capital goods may be impossible to sell), and from capital specificity (see Dixit and Pindyck, 1994, and Trigeorgis, 1996) ${ }^{3}$. The irreversibility of capital expenditures is even more obvious in markets subject to price regulation, which are typically natural monopolies; the scarcity (or total absence) of firms operating in the same sector and the public constraints coming from nature of the service may represent decisive factors in this respect $^{4}$. Relative to this literature, we consider how investment is affected by different regulatory rules, showing how the option value of irreversible investments matters in determining the optimal regulatory policy.

The second stream of literature is the one on regulation and investment. Since Laffont and Tirole (1986), we know that optimal price schemes entail a distortion in firms' choices. To tackle this problem, the rule labelled " $R P I-$ $x$ " was introduced. In this case, investment does not affect price, so that this rule is supposed to have "minimum" distortionary effects on investment choices.

Beesley and Littlechild (1989) stress that the efficiency properties of this

important characteristics, investment irreversibility, uncertainty and the ability to choose the optimal timing of investment".

${ }^{3}$ Irreversibility may be caused by industry comovements as well: when a firm wants to resell its capital because of negative market conditions, but potential buyers operating in the same industry are subject to the same conditions, the firm may have to sell the capital at a lower price than otherwise possible.

${ }^{4} \mathrm{An}$ idea of the empirical relevance of irreversible investments in regulated industries can be obtained looking at the so called "stranded costs", i.e., at the value of assets that following liberalisation will hardly find a remuneration, but cannot be shifted to a different productive use. According to Lyon and Mayo (2000) these costs can be estimated for the US electricity sector "in the neighborhood of $\$ 200$ billion". 
scheme may be undermined by two aspects. The first one is that the $x$ factor is subject to periodic reviews (every $4-5$ years), so that a cost decrease may be exploited by the regulator to decrease prices in the future. The second factor is the risk of expropriation, which means that, even before the review, the regulator may be tempted to intervene - following political pressure - in case firm's profits appear as "excessively" high.

In our paper we consider the option value of an investment of a given amount, to see how different regulatory schemes affect the timing of investment. Notice that here we do not introduce any of these elements indicated by Beesley and Littlechild (1989) as countervailing factors, so that one would expect $R P I-x$ to emerge as the more desirable regulatory rule. On the contrary, our main result is that, even in the extremely favorable case we examine, profit sharing does not underperform the purest version of $R P I-x .^{5}$

The paper is organized as follows. The next section provides the analytical set-up in continuous time, stressing how profit sharing does not reduce the incentive to invest relative to a pure price-cap scheme when lump-sum irreversible investments are considered. Section 3 extends the model to the case of cost-reducing investments, showing how the same result follows. Section 4 concludes the paper.

\section{The model}

In this section we introduce a simple continuous-time infinite horizon model describing the behavior of a firm. The market is characterized by the demand function $q(t)=q(p(t))$, where $p$ and $q$ are price and quantity of the good at time $t$. Production takes place at a per-period cost given by $C=c(t) q(t)$. Furthermore, in order to produce the firm needs to build an infrastructure:

- Assumption 1 (investment). Production requires a one-off investment of a given amount $I$.

This could be the case, for instance, of an energy distributor that has to decide whether or not to invest in a new network (either a pipeline or wires) in order to serve a town. A relevant aspect of this assumption is that the amount of investments is given. Although firms often have the possibility to

\footnotetext{
${ }^{5}$ This result can be usefully linked to some recent results of the empirical literature. For instance, Crew and Kleindorfer (1996) and the papers they review stress how the presumed superiority of optimal price rules does not emerge so clearly from experiences in different countries and sectors. The claim that $R P I-x$ rules lead to more efficient investment patterns than profit related regulatory schemes does not find a clear empirical support.
} 
marginally adjust the value of their expenditures, it is also true that the size of most investment projects that utilities face is by and large determined by the size of the area they want to serve. Building a new electric line connecting two nodes of a transmission system to improve its reliability, or a pipeline to sell gas to a new city are choices that entail an expenditure that can only partially be controlled by the firm. This type of major investments is what we focus on ${ }^{6}$.

In these cases the firm is left with two major choices: whether or not to undertake the investment, and when to do so. Therefore, while the notion of "underinvestment" typically refers to the amount spent by the firm, in this context we will talk of underinvestment referring to the probability that a firm invests and to the date of the investment, i.e. to the present expected value of the investment. The apparent difference between our notion and the usual one is simply due to the fact that we explicitly model uncertainty and time.

If the firm does not undertake the investment, it cannot produce and its profit is zero. If the firm invests, per-period profits are ${ }^{7}$

$$
\Pi(t)=[p(t)-c(t)] q(t)
$$

The firm has an infinite time horizon and maximizes the (discounted) present value of future expected profit.

Demand is stochastic, and we introduce the following

- Assumption 2. Demand follows a geometric Brownian motion

$$
d q(t)=\alpha_{q} q(t) d t+\sigma_{q} q(t) d z_{q}
$$

where $\alpha_{q}$ and $\sigma_{q}$ are the growth rate and variance parameter, respectively.

Cost follows the dynamics given by

$$
c(t)=c_{0} e^{-\gamma t}
$$

where the parameter $\gamma \geq 0$ captures possible cost reductions over time. Price is determined by the regulator in a way we will define in the next sections.

\footnotetext{
${ }^{6}$ According to Leahy (1993), however, nothing substantial changes if we focus on investment of endogenous size. We explore this issue in a companion paper where irreversible quality choices of endogenous size are considered (Panteghini and Scarpa, 2002).

${ }^{7}$ As we look at the consequences of different regulatory schemes on a firm's decisions, costs are considered known. The choice of the optimal price should instead consider asymmetric information, but this is beyond the scope of the current work.
} 


\subsection{Pure price cap}

We will first assume that price regulation follows the traditional price-cap rule known as $R P I-x$ (Beesley and Littlechild, 1989).

Definition 1 (Price Cap) Under the Price Cap RPI - x rule, if the firm starts producing at time $t^{*}$, the initial price $p_{0}$ is given, and its dynamics is defined by the difference between the inflation rate (changes in the retail price index, RPI) and an exogenous factor $x$ :

$$
p(t)=p_{0} e^{(R P I-x) t}
$$

for $t \geq t^{*}$.

The factor $x$ is linked to the productivity gain (cost reduction) that the regulator expects the firm to be capable of achieving every year, but is determined at the beginning and is thus exogenous to the firm. As already stressed, the logic of the $R P I-x$ rule is that, by making prices insensitive at the margin to firm's choices, it appears to eliminate underinvestment. Also notice that here we assume that price dynamics is given over an infinite horizon, so that current investments have no impact on prices either in the shortor in the long-run.

For simplicity we assume that $[p(t)-c(t)]$ has the following dynamics ${ }^{8}$

$$
\frac{d[p(t)-c(t)]}{d t}=[p(t)-c(t)](R P I-x+\gamma)
$$

Using equations (1), (3), and (2), and applying Itô's lemma we can obtain the profits' dynamics

$$
d \Pi(t)=\alpha \Pi(t) d t+\sigma \Pi(t) d z
$$

${ }^{8}$ The assumed dynamics of $[p(t)-c(t)]$ is necessary for obtaining a closed-form solution. Equation (3) is a special case of a more general formulation obtained by assuming that price and cost evolve according to $p(t)=p_{0} e^{(R P I-x) t}$ and $c(t)=c_{0} e^{-\gamma t}$

If we use the above equations and differentiate $[p(t)-c(t)]$, we obtain

$$
\begin{aligned}
\frac{d[p(t)-c(t)]}{d t}= & \{(R P I+\gamma-x)[p(t)-c(t)]+ \\
& (R P I+\gamma-x) c(t)-\gamma[p(t)-c(t)]\}
\end{aligned}
$$

Setting $\{(R P I+\gamma-x) c(t)-\gamma[p(t)-c(t)]\}=0$ we obtain (3). Note that the above equality implies that the mark-up is constant, i.e.

$$
\frac{p(t)-c(t)}{c(t)}=\frac{R P I+\gamma-x}{\gamma}
$$


where $\alpha \equiv R P I-x+\gamma+\alpha_{q}$ is the expected growth rate of per-period profits and $\sigma=\sigma_{q}$ is the standard deviation ${ }^{9}$. Given the dividend rate $\delta$ (which must be positive in order for the net value of the firm to be bounded) and the risk-free interest rate $r$, we must have $r-\delta=\alpha^{10}$. Solving for the dividend rate we thus obtain

$$
\delta(x)=r-R P I+x-\alpha_{q}-\gamma
$$

The firm must solve a standard optimal stopping time problem, namely it must choose the timing of investment to maximize the expected present value of its payoff. The problem can be represented as follows

$$
\max _{t} E\left[\left(V_{P C}(\Pi(t))-I\right) e^{-r t}\right]
$$

where $E[$.$] denotes the expectation operator, V_{P C}(\Pi(t))$ is the project value under the price-cap, i.e. the $N P V$ of the project at time $t$. The solution of the problem, i.e. the optimal time of investments, will be defined as $t^{*}$.

Using dynamic programming, the firm's value $V_{P C}(\Pi(t))$ can be written as

$$
V_{P C}(\Pi(t))=\Pi(t) d t+e^{-r d t} E\left[V_{P C}(\Pi(t)+d \Pi(t))\right]
$$

Expanding the right-hand side and using Itô's lemma one obtains

$$
r V_{P C}(\Pi(t))=\Pi(t)+(r-\delta(x)) \Pi V_{P C_{\Pi}}(\Pi(t))+\frac{\sigma^{2}}{2} \Pi^{2} V_{P C_{\Pi \Pi}}(\Pi(t))
$$

where $V_{P C_{\Pi}}=\partial V_{P C} / \partial \Pi(t)$ and $V_{P C_{\Pi \Pi}}=\partial^{2} V_{P C} / \partial \Pi^{2}(t)$, respectively. For simplicity, hereafter, we will omit the time variable $t$.

To compute the value function, it is assumed that $V_{P C}(0, x)=0$, namely when $\Pi$ is very small, the project is almost worthless, and that no speculative bubbles exist ${ }^{11}$. Thus, equation (7) has the following solution

$$
V_{P C}(\Pi, x)=\Pi / \delta(x)
$$

\footnotetext{
${ }^{9}$ The model could be easily extended by assuming that $[p(t)-c(t)]$ follows a stochastic process, i.e.

$$
d[p(t)-c(t)]=[p(t)-c(t)]\left[(R P I+\gamma-x) d t+\sigma_{p c} d z_{p c}\right] .
$$

This may be the case, for instance, if the $R P I$ is a random variable and if technology shocks may take place.

${ }^{10}$ As shown in Panteghini and Scarpa (2001) considering shareholders' risk aversion does not change the result.

${ }^{11}$ See Dixit and Pindyck (1994, Ch. 5 and 6).
} 
As shown by Dixit and Pindyck (1994), the option function has the following form

$$
O_{P C}(\Pi, x)=A \Pi^{\beta_{1}(x)}
$$

where $A$ is a parameter to be determined, and $\beta_{1}(x)$ is the positive root of the following characteristic equation ${ }^{12}$

$$
\frac{\sigma^{2}}{2} \beta(\beta-1)+(r-\delta(x)) \beta-r=0
$$

The optimal investment timing can be computed using the Value Matching Condition $(V M C)$ and the Smooth Pasting Condition $(S P C)$. The former condition requires the net present value of the project to be equal to the option value to defer investment, $O_{P C}(\Pi, x)$, namely

$$
V_{P C}(\Pi, x)-I=O_{P C}(\Pi, x)
$$

The second condition requires the slopes of the functions $\left[V_{P C}(\Pi, x)-I\right]$ and $O_{P C}(\Pi, x)$ to match

$$
\frac{\partial\left[V_{P C}(\Pi, x)-I\right]}{\partial \Pi}=\frac{\partial O_{P C}(\Pi, x)}{\partial \Pi}
$$

Conditions $V M C$ and $S P C$ characterize optimal time $t^{*}$. Notice that, given (4), this value can be associated to a profit level $\Pi^{*}$ : whenever current profit reaches $\Pi^{*}$, the firm invests.

To solve the optimal stopping time problem, let us substitute (8) and (9) into the $V M C$ and the $S P C$. We thus obtain a two-equation system with two unknowns: the trigger point of $\Pi$, above which investment is profitable, and the coefficient $A$. It is easy to show that the trigger point is ${ }^{13}$

$$
\Pi_{P C}^{*}(x) \equiv \frac{\beta_{1}(x)}{\beta_{1}(x)-1} \delta(x) I
$$

${ }^{12}$ The positive root is

$$
\beta_{1}(x)=\frac{1}{2}-\frac{r-\delta(x)}{\sigma^{2}}+\sqrt{\left(\frac{1}{2}-\frac{r-\delta(x)}{\sigma^{2}}\right)^{2}+\frac{2 r}{\sigma^{2}}} .
$$

It is easy to ascertain that $\frac{\partial \beta_{1}(x)}{\partial x}>0$.

${ }^{13}$ Substituting $\Pi_{P C}^{*}(x)$ into the system one easily obtains

$$
A=\frac{I}{\beta_{1}(x)-1}\left(\Pi_{P C}^{*}(x)\right)^{-\beta_{1}(x)}>0
$$


The option value multiple in equation $(10), \frac{\beta_{1}(x)}{\beta_{1}(x)-1}>1$, shows that the gross present value

$$
V_{P C}^{*}\left(\Pi_{P C}^{*}(x), x\right) \equiv \frac{\Pi_{P C}^{*}(x)}{\delta(x)}=\frac{\beta_{1}(x)}{\beta_{1}(x)-1} I
$$

must exceed the investment cost $I$ to compensate for irreversibility. Given $\frac{\partial \beta_{1}(x)}{\partial x}>0, \frac{\partial\left(\frac{\beta_{1}(x)}{\beta_{1}(x)-1}\right)}{\partial x}<0$. This means that an increase in $x$ decreases both the expected profit $V_{P C}^{*}$ and - given the $(V M C)$ - the opportunity cost of investing $O_{P C}^{*}$.

As shown by Dixit and Pindyck (1994), who provide some numerical simulations, for reasonable values of the parameters, an increase in $\alpha$ raises the trigger point, so that $\frac{\partial \Pi_{P C}^{*}(x)}{\partial x}>0$, i.e., an increase in the $x$ factor increases the option function more than the value function, thereby tending to postpone investment.

Remark 1 An increase in $x$ increases $\Pi_{P C}^{*}$ (i.e., $\left.\frac{\partial \Pi_{P C}^{*}}{\partial x}>0\right)$.

This implies that having a $R P I-x$ reduces the incentive to invest. Therefore, the $R P I-x$ rule is not neutral to investment decisions, a claim by Beesley and Littlechild (1989).

Remark 2 We can label the above result "underinvestment" in that, given initial price and a distribution of cost parameters, the present expected value of investment is reduced because of the Price Cap rule.

\subsection{Profit sharing}

The $R P I-x$ rule has been criticised on the ground that cost decreases are often more substantial than predicted, and this leaves the firm most of the increase in surplus which follows privatisation.

To tackle this fairness concern in a predictable way, an alternative to $R P I-x$ has been proposed, called profit sharing ${ }^{14}$. This scheme is defined as follows:

Definition 2 (Profit sharing) Under the Profit Sharing regulatory mechanism, the $R P I-x$ rule remains in place as long as profit remains below

\footnotetext{
${ }^{14}$ Notice that one could also have an intervention rule based on the level of revenues instead of profits; see Sappington and Weisman (1996).
} 
an exogenous level $\widetilde{\Pi}$. If $\Pi(t)>\widetilde{\Pi}$, the $x$ factor immediately ${ }^{15}$ increases to $x^{\prime}>x$ :

$$
p(t)= \begin{cases}p_{0} e^{(R P I-x) t} & \text { if } \Pi(t) \leq \widetilde{\Pi} \\ p_{0} e^{\left(R P I-x^{\prime}\right) t} & \text { if } \Pi(t)>\widetilde{\Pi}, \text { with } x^{\prime}>x\end{cases}
$$

Thus, the price decrease factor remains constant as long as profits are considered "reasonable". When they become "excessive", this mechanism re-distributes part of the surplus to the consumers. In this section we analyze this issue.

If $\Pi<\widetilde{\Pi}$ the Brownian motion is the same as in the previous section. Notice that it is natural to assume that the $\widetilde{\Pi}$ is above the trigger point. Otherwise, the price scheme would start from a value of $x$ already equal to $x^{\prime}$. This would obviously contradict the definition of profit sharing, i.e. the idea that regulation starts with a given value of $x$, which is made more stringent at a later stage, in case profit goes beyond a certain level.

If, instead, $\Pi>\widetilde{\Pi}$, the Brownian motion describing the regulated payoff is $^{16}$

$$
d \Pi=\alpha^{\prime} \Pi d t+\sigma \Pi d z
$$

with $\alpha^{\prime} \equiv R P I-x^{\prime}+\gamma+\alpha_{q}<\alpha$. If $\Pi>\widetilde{\Pi}$, therefore, the dividend rate is given by equality $r-\delta\left(x^{\prime}\right)=\alpha^{\prime}-\lambda \sigma \rho_{M}$, which implies the inequalities $\delta\left(x^{\prime}\right)>\delta(x)>\delta$. When a switch point $\widetilde{\Pi}$ is introduced, both the option function and the value function must be solved separately for $\Pi<\widetilde{\Pi}$ and $\Pi>\widetilde{\Pi}$. Then, the values and derivatives of the functions are equated at the switch point $\Pi=\widetilde{\Pi}$ (see Dixit and Pindyck, 1994, pp. 186-189).

In order to check whether an investment project is profitable, both explicit and opportunity costs must be taken into account. Thus, investment is profitable if (and when) the present discounted value of future profits, net of both costs, is positive.

Following the same procedure as above, we start with the analysis of the value function. The general solution is given by the sum of a perpetual rent, with discount rate $\delta(x)$, and a homogeneous (exponential) part. Again, it is

\footnotetext{
${ }^{15}$ In a discrete-time framework it would be sensible to introduce a delay between the observation of a profit level and the adjustment of the $x$ factor. In this set-up this would introduce a very substantial analytical complication with no relevant change in the results.

${ }^{16}$ Notice that it may well happen that profit first goes beyond $\widetilde{\Pi}$, while at a later stage $\Pi<\widetilde{\Pi}$. In this case - in line with the spirit of the mechanism at stake - this formulation guarantees that the price cap goes back to its original level.
} 
assumed that $V_{P S}(0, x)=0$ and that no speculative bubbles exist. Thus the solution of the value function is

$$
V_{P S}(\Pi, x)= \begin{cases}\frac{\Pi}{\delta(x)}+V_{1} \Pi^{\beta_{1}(x)} & \text { if } \quad \Pi<\widetilde{\Pi} \\ \frac{\Pi}{\delta\left(x^{\prime}\right)}+V_{2} \Pi^{\beta_{2}\left(x^{\prime}\right)} & \text { if } \quad \Pi>\widetilde{\Pi}\end{cases}
$$

As shown in Panteghini and Scarpa (2001), equating the two components of (12) at the switch point $\Pi=\widetilde{\Pi}$ and considering the $S P C$ one obtains parameters $V_{1}$ and $V_{2}$. Both parameters depend on the regulatory coefficients $x$ and $x^{\prime}$. In particular, $V_{1} \Pi^{\beta_{1}(x)}<0$ : this represents the present value of future profit changes due to the profit sharing (when $\Pi$ goes beyond $\widetilde{\Pi}$ ). $V_{2} \Pi^{\beta_{2}\left(x^{\prime}\right)}>0$ measures the present value of the future increase in the profit participation when $\Pi$ goes below $\widetilde{\Pi}$ (in fact $\partial \Pi^{\beta_{2}\left(x^{\prime}\right)} / \partial \Pi<0$ ).

Let us now turn to the option value, $O_{P S}(\Pi, x)$. In the $(0, \widetilde{\Pi})$ region, condition $O_{P S}(0, x)=0$ holds, and, therefore, the value function has the standard form $C_{1} \Pi^{\beta_{1}(x)}$. In the $(\widetilde{\Pi}, \infty)$ region, instead, the option function is given by the sum of $B_{1} \Pi^{\beta_{1}\left(x^{\prime}\right)}$ and $B_{2} \Pi^{\beta_{2}\left(x^{\prime}\right)}$ (with $B_{1}$ and $B_{2}$ to be determined). $\beta_{1}\left(x^{\prime}\right)$ and $\beta_{2}\left(x^{\prime}\right)$ are the roots of the characteristic equation $\frac{\sigma^{2}}{2} \beta(\beta-1)+\left(r-\delta\left(x^{\prime}\right)\right) \beta-r=0$, with $\beta_{1}\left(x^{\prime}\right)>1$ and $\beta_{2}\left(x^{\prime}\right)<0^{17}$. To sum up, the option function is

$$
O_{P S}(\Pi, x)= \begin{cases}C_{1} \Pi^{\beta_{1}(x)} & \text { if } \Pi<\widetilde{\Pi} \\ B_{1} \Pi^{\beta_{1}\left(x^{\prime}\right)}+B_{2} \Pi^{\beta_{2}\left(x^{\prime}\right)} & \text { if } \Pi>\widetilde{\Pi}\end{cases}
$$

By equating the values and the derivatives of the two components of the option function at point $\Pi=\widetilde{\Pi}$, we can compute $B_{1}$ and $B_{2}$ as functions of $C_{1}$. As shown in Panteghini and Scarpa (2001), $B_{1} \propto C_{1}$ and $B_{2} \propto C_{1}$.

Substituting equations (13) and (12) into the $V M C$ and $S P C$ one obtains the trigger point and the unknown parameter $C_{1}$ of the option function. In the $(0, \widetilde{\Pi})$ region, these conditions lead to the following system

$$
\begin{gathered}
\frac{\Pi}{\delta(x)}+V_{1} \Pi^{\beta_{1}(x)}-I=C_{1} \Pi^{\beta_{1}(x)} \\
\frac{1}{\delta(x)}+V_{1} \beta_{1}(x) \Pi^{\beta_{1}(x)-1}=C_{1} \beta_{1}(x) \Pi^{\beta_{1}(x)-1}
\end{gathered}
$$

${ }^{17}$ The roots are

$$
\beta_{1,2}\left(x^{\prime}\right)=\frac{1}{2}-\frac{r-\delta\left(x^{\prime}\right)}{\sigma^{2}} \pm \sqrt{\left(\frac{1}{2}-\frac{r-\delta\left(x^{\prime}\right)}{\sigma^{2}}\right)^{2}+\frac{2 r}{\sigma^{2}}},
$$

and it is easy to ascertain that, given derivative $\frac{\partial \beta_{1}(x)}{\partial x}>0$, inequality $\beta_{1}\left(x^{\prime}\right)>\beta_{1}(x)$ holds. 
which yields the same trigger point as the one obtained under the pure pricecap system in equation $(10)^{18}$

$$
\Pi_{P S}^{*}(x) \equiv \frac{\beta_{1}(x)}{\beta_{1}(x)-1} \delta(x) I,
$$

The equality between $\Pi_{P S}^{*}(x)$ and $\Pi_{P C}^{*}(x)$ establishes the following:

Proposition 1 (Neutrality of profit sharing) Consider a regulated monopolist which has to decide on an investment of a given amount. When demand is uncertain as modelled in (2) and the timing of investment is endogenous, correcting the $R P I-x$ rule with a profit sharing element does not affect the timing of investment.

The neutrality (indifference) result can be explained as follows.

Since the tightening of the price cap takes place only in case of "good news", the bad news principle implies that, while profit sharing actually reduces the firm's rents, it does not interfere with its decision to invest relative to the pure price-cap rule. There are no investment projects that will be undertaken under one regime, but not under the other.

Another way to look at the issue is to stress that profit sharing is equivalent to equity participation by the consumers. Recall, in fact, that when $\Pi>\widetilde{\Pi}$, a given part of the surplus is redistributed to the consumers. When

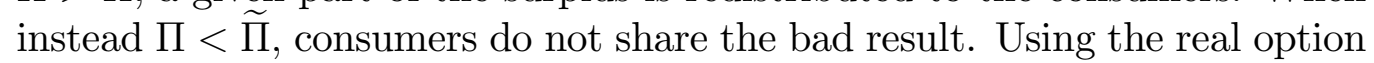
approach we can thus say that the profit sharing device is equivalent to a case where consumers are endowed with a put option with strike price $\widetilde{\Pi}$, written on the firm's profits. If, therefore, the firm's return drops below $\widetilde{\Pi}$ (bad result), consumers sell their equity participation at zero price. Then, they will re-buy (at zero price) their participation when the firm faces a good result, namely when $\Pi>\widetilde{\Pi}$.

To clarify this point, let us concentrate on the $(0, \widetilde{\Pi})$ region $\left(\right.$ with $\Pi_{P S}^{*}(x)<$ $\widetilde{\Pi})$, and recall equations (13), (12) and the solution of $C_{1}$. The negative term $V_{1} \Pi^{\beta_{1}(x)}$ measures the value of the consumers' put option, which must be added to both the project value and the option function. This addition is necessary because, irrespective of whether the firm is waiting or producing, a worthy put option is owned by the consumers. Since $V_{1} \Pi^{\beta_{1}(x)}$ enters both

\footnotetext{
${ }^{18} \mathrm{It}$ is easy to show that

$$
C_{1}=\frac{\beta_{2}\left(x^{\prime}\right)-1}{\beta_{1}(x)-\beta_{2}\left(x^{\prime}\right)} \frac{\delta\left(x^{\prime}\right)-\delta(x)}{\delta\left(x^{\prime}\right) \delta(x)} \widetilde{\Pi}^{1-\beta_{1}(x)}+\frac{1}{\beta_{1}(x)} \frac{\Pi_{P S}^{*}(x)^{1-\beta_{1}(x)}}{\delta(x)}>0 .
$$
}


functions, the difference $\left[V_{P S}(\Pi, x)-O_{P S}(\Pi, x)\right]$ is independent of the switch level $\widetilde{\Pi}$, thereby making the profit-sharing device neutral.

Easy computations show that, for a given payoff, the higher the switch point the greater the value of $V_{1}$. This implies that $\frac{\partial C_{1}}{\partial \widetilde{\Pi}}=\frac{\partial V_{1}}{\partial \widetilde{\Pi}}>0$ (with $\left.V_{1}<0\right)$. Therefore, an increase in $\widetilde{\Pi}$ raises both the value and the option function by the same amount, and vice versa. Finally, note that the higher the switch point $\widetilde{\Pi}$ the lower the put option value $\left(-V_{1} \Pi^{\beta_{1}(x)}\right)$. Of course, for $\widetilde{\Pi} \rightarrow \infty$, profit sharing vanishes, and the put option turns to be nil.

\section{Incremental investment and cost reduction}

So far, we have considered investments which are necessary to produce. A second category of investment is at least as relevant (and analyzed in the literature), i.e. cost-reducing investments. In this section we want to extend the previous framework to accommodate for the latter category, showing how the same results follow. To this end, we modify Assumption 1 in the following way:

- Assumption 1' (investment). Production requires a one-off investment of a given amount $I_{1}$, that is undertaken at time 0 . At any time after $t^{*} \geq 0$, the firm has the possibility to invest a further amount $I_{2}$, which reduces its variable cost.

The firm can wait to invest $I_{2}$ until the current payoff is sufficiently high. By investing $I_{1}$, the firm receives a per-period payoff $\Pi$ and acquires an (American call) option to invest again. When it undertakes investment $I_{2}$, variable profits increase.

The cost reduction can be modelled as a downward jump in operating costs. For simplicity we assume that the after-investment profit margin $\left[p(t)-c^{\prime}(t)\right]$ follows the same dynamics as $[p(t)-c(t)]$, namely

$$
\Pi(t)=\left[p(t)-c^{\prime}(t)\right] q(t)=\Psi(t)[p(t)-c(t)] q(t)
$$

where $\Psi(t)$ is

$$
\Psi(t)=\left\{\begin{array}{c}
1 \text { for } t<T \\
\Psi \geq 1 \text { for } t>T
\end{array}\right.
$$

and $T$ is the optimal time of investment $I_{2}$, to be determined. Notice that (17) captures the idea that the second investment reduces costs, but a similar story could be told if investment contributes to expand revenues even further. 


\subsection{Price cap}

Let us now compute the firm's value function. Recall equation (7). When investment $I_{1}$ is undertaken, given the boundary condition $V_{P C}^{1}(0, x)=0$, the value function has a standard solution

$$
V_{P C}^{1}(\Pi, x)=\frac{\Pi}{\delta(x)}+E_{1} \Pi^{\beta_{1}(x)}
$$

Namely, the firm's project is given by the perpetual rent $\Pi / \delta(x)$ plus the term $E_{1} \Pi^{\beta_{1}(x)}$, which measures the value of the option to invest $I_{2}$. Thus, the firm may find it profitable to invest if the net present value of investment $I_{1}$ is positive, i.e. $V_{P C}^{1}(\Pi, x)>I_{1}$. Therefore, the firm may decide to enter this market even if the present value of its current profits is negative, i.e. $\frac{\Pi}{\delta(x)}<I_{1}$.

When the firm invests $I_{2}$, per-period profits jump upward and the firm's project becomes $V_{P C}^{2}(\Pi, x)$. Since, by assumption, there are no bubbles and condition $V_{P C}^{2}(0, x)=0$ holds, the firm's value is simply a perpetual rent

$$
V_{P C}^{2}(\Pi, x)=\frac{\Psi \Pi}{\delta(x)}>\frac{\Pi}{\delta(x)}
$$

Given equations (18) and (19), one can compute the trigger point above which incremental investment is profitable. On the one hand, investment $I_{2}$ increases profits. On the other hand, it entails the loss of the perpetual rent earned with investment $I_{1}$ and the exercise of the call option. Thus, investment is profitable if the firm's project value is at least equal to the sum of the explicit cost $I_{2}$ and the opportunity cost $V_{P C}^{1}(\Pi, x)$. In this case, therefore, the $V M C$ and $S P C$ define a two-equation system

$$
\begin{gathered}
V_{P C}^{1}(\Pi, x)=V_{P C}^{2}(\Pi, x)-I_{2} \\
\frac{\partial V_{P C}^{1}(\Pi, x)}{\partial \Pi}=\frac{\partial V_{P C}^{2}(\Pi, x)}{\partial \Pi}
\end{gathered}
$$

where the trigger point $\Pi_{P C}^{*^{\prime}}$ and parameter $E_{1}$ are the unknowns. Solving the system in the same way already employed yields ${ }^{19}$

$$
\Pi_{P C}^{*^{\prime}}=\frac{\beta_{1}(x)}{\beta_{1}(x)-1} \frac{\delta(x)}{\Psi-1} I_{2}
$$

\footnotetext{
${ }^{19}$ The unknown parameter is equal to

$$
E_{1}=\frac{\Psi-1}{\beta_{1}(x) \delta(x)} \cdot \Pi_{P C}^{*^{1-\beta_{1}(x)}}>0 .
$$

Notice that $\frac{\partial E_{1}}{\partial \Psi}>0$, namely the greater the increase in profitability, the more valuable the option to reduce costs is.
} 


\subsection{Profit sharing}

Let us now turn to the Profit Sharing rule. If $\Pi<\widetilde{\Pi}$ the Brownian motion is the same as in the previous section. In the $(0, \widetilde{\Pi})$ region, the firm's project is given by the perpetual rent $\Pi / \delta(x)$ plus a term $F_{1} \Pi^{\beta_{1}(x)}$, which measures the value of the option to reduce costs. In the $(\widetilde{\Pi}, \infty)$ region, the value function has the general form, namely it is given by the sum of $G_{1} \Pi^{\beta_{1}\left(x^{\prime}\right)}$ and $G_{2} \Pi^{\beta_{2}\left(x^{\prime}\right)}$ (with $G_{1}$ and $G_{2}$ to be determined). To sum up, the value function is

$$
V_{P S}^{1}(\Pi, x)=\left\{\begin{array}{l}
\frac{\Pi}{\delta(x)}+F_{1} \Pi^{\beta_{1}(x)} \quad \text { if } \Pi<\widetilde{\Pi} \\
\frac{\Pi}{\delta(x)}+G_{1} \Pi^{\beta_{1}\left(x^{\prime}\right)}+G_{2} \Pi^{\beta_{2}\left(x^{\prime}\right)} \quad \text { if } \quad \Pi>\widetilde{\Pi}
\end{array}\right.
$$

By equating the values and the derivatives of the two components of the option function at point $\Pi=\widetilde{\Pi}$, we can compute $G_{1}$ and $G_{2}$. It is possible to show that $G_{1} \propto F_{1}$ and $G_{2} \propto F_{1}$.

Let us now turn to the value function after investing $I_{2}$. The general solution of the value function is given by the sum of a perpetual rent, with discount rate $\delta(x)$, and a homogeneous (exponential) part. Again, to compute the value function, it is assumed that $V_{P S}^{2}(0, x)=0$ (namely when $\Pi$ is very small, the project is almost worthless) and that no speculative bubbles exist. The function thus reduces to

$$
V_{P S}^{2}(\Pi, x)= \begin{cases}\frac{\Psi \Pi}{\delta(x)}+L_{1} \Pi^{\beta_{1}(x)} & \text { if } \quad \Pi<\widetilde{\Pi} \\ \frac{\Psi \Pi}{\delta\left(x^{\prime}\right)}+L_{2} \Pi^{\beta_{2}\left(x^{\prime}\right)} & \text { if } \quad \Pi>\widetilde{\Pi}\end{cases}
$$

As shown in Panteghini and Scarpa (2001), $L_{1}=\Psi V_{1}<0$ and $L_{2}=\Psi V_{2}>0$. Substituting equations (21) and (22) into the $V M C$ and $S P C$ one obtains the trigger point and the unknown parameter $F_{1}$. In the $(0, \widetilde{\Pi})$ region, these conditions lead to the following system

$$
\begin{gathered}
\frac{\Psi \Pi}{\delta(x)}+L_{1} \Pi^{\beta_{1}(x)}-I_{2}=\frac{\Pi}{\delta(x)}+F_{1} \Pi^{\beta_{1}(x)} \\
\frac{\Psi}{\delta(x)}+L_{1} \beta_{1}(x) \Pi^{\beta_{1}(x)-1}=\frac{1}{\delta(x)}+F_{1} \beta_{1}(x) \Pi^{\beta_{1}(x)-1}
\end{gathered}
$$

which yields (20)

$$
\Pi_{P S}^{*^{\prime}}=\frac{\beta_{1}(x)}{\beta_{1}(x)-1} \cdot \frac{\delta(x)}{\Psi-1} \cdot I_{2}
$$


Comparing conditions (20) and (23), it appears that ${ }^{20}$

$$
\Pi_{P C}^{*^{\prime}}=\Pi_{P S}^{*^{\prime}}
$$

This can be summarized in the following way:

Proposition 2 (Neutrality of profit sharing on cost-reducing investment) Consider a regulated monopolist which has to decide on a cost-reducing investment of a given amount. When demand is uncertain as modelled in (2) and the timing of cost-reducing investment is endogenous, correcting the $R P I-x$ rule with a profit sharing element does not affect the timing of investment.

This result simply reflects the parallel between loosening the price-cap and reducing costs. Both events represent "good news" and the endogeneity of the decision to reduce costs does not affect the neutrality result developed in the previous section.

\section{Conclusions}

Relative to the existing literature, which implicitly assumed reversible investment by regulated firms, our results appear significantly different. While current literature indicates that profit-sharing has a negative effect on investment decisions, our paper shows that this is not true. What makes a difference is the introduction of two fairly realistic assumptions: investment irreversibility and the firm's ability to decide when undertaking it. This implies that the firm is endowed with a call option to delay, which expires when investment is undertaken. We have thus shown that a profit-sharing device reduces both the value of the project and the value of the option to wait by the same amount. According to the Bad News Principle, therefore, no additional distortion is introduced, with respect to price cap.

Moreover, it is worth noting that one of the regulators' main targets is the rent extraction per se. After all, the very notion of profit-sharing comes from the idea that a scheme which yields an excessively imbalanced distribution of rents is undesirable, and the rate of return regulation scheme still prevailing

${ }^{20}$ Easy computations show that

$$
F_{1}=L_{1}+\frac{1}{\beta_{1}(x)} \frac{\Pi_{P S}^{*^{\prime}}(x)^{1-\beta_{1}(x)}}{\delta(x)}
$$


in a large part of the US is based on the idea that restraining monopoly rents is a goal by itself. What we show here, therefore, has an important policy implication. Since profit sharing has a greater ability to raise rents than price cap, but does not cause any additional distortion, it is possible to extract the same amount of rents with a lower value of $x$. Given the amount of rents extracted from the monopolist, under profit sharing the trigger point above which investment is profitable is thus lower than under a pure price cap; in other words, investment is undertaken earlier than under the pure price cap regime.

A question that still remains open, is the role that political uncertainty plays. One of the reasons why profit sharing has been proposed is that regulatory authorities are unable to commit not to intervene if the regulated firm's profits turn out to be very high. By automatically curbing profits, a "sliding scale" device might decrease the regulator's incentive to intervene, thereby increasing the firm's incentive to invest. This element of "political risk" could be included in the model we have developed, by interpreting the stochastic disturbance on profit as a regulation induced disturbance, but a more satisfactory characterisation of political intervention - considering the $x$ factor as a stochastic element - is an issue that we leave for future investigation.

\section{References}

[1] Abel A.B., A.K. Dixit, J.C. Eberly and R.S. Pindyck (1996), Options, the Value of Capital, and Investment, Quarterly Journal of Economics, v. 111, iss. 3, pp. 753-77.

[2] Armstrong, M. S. Cowan and J. Vickers (1995), Regulatory Reform. Economic Analysis and British Experience, MIT Press, Cambridge, Mass.

[3] Beesley, M. and S. Littlechild (1989), The Regulation of Privatized Monopolies in the United Kingdom, Rand Journal of Economics, 20, 454-72.

[4] Bernanke, B.S. (1983), Irreversibility, Uncertainty, and Cyclical Investment, Quarterly Journal of Economics, 98, 85-103.

[5] Burns, P., R. Turvey and T. G. Weyman-Jones (1998), The Behavior of the Firm under Alternative Regulatory Constraints, Scottish Journal of Political Economy, 45(2), 133-57 
[6] Crew, M. and P. Kleindorfer (1996), Incentive Regulation in the United Kingdom and the United States: Some Lessons, Journal of Regulatory Economics, 9, 211-25.

[7] Dixit, A. and Pindyck R.S. (1994), Investment under Uncertainty, Princeton University Press.

[8] Karlin S. and H.M. Taylor (1975), A First Course in Stochastic Processes, New York, Academic Press.

[9] Laffont, J. J. and J. Tirole (1986), Using Cost Observations to Regulate Firms, Journal of Political Economy, vol.94, 614-41.

[10] Leahy, J. P. (1993), Investment in Competitive Equilibrium: the Optimality of Myopic Behavior, Quarterly Journal of Economics, 108, 11051133 .

[11] Lyon, T. (1996), A Model of Sliding-Scale Regulation, Journal of Regulatory Economics, 9, 227-47.

[12] Lyon, T. and J. Mayo (2000), Regulatory Opportunism and Investment behavior: Evidence from the U.S. Electricity Utility Industry, Indiana University Discussion Paper.

[13] Mayer, C. and J. Vickers (1996), Profit-Sharing Regulation: An Economic Appraisal, Fiscal Studies, 17, 1-18.

[14] Panteghini, P. and C. Scarpa (2001), Incentives to (irreversible) investments under different regulatory regimes, CES-ifo Working Paper No. 417.

[15] Panteghini, P. and C. Scarpa (2002), Endogenous incremental investments and price regulation, mimeo, University of Brescia.

[16] Sappington, D. and D. Weisman (1996), Revenue Sharing in Incentive Regulation Plans, Information Economics and Policy, 8, 229-48.

[17] Trigeorgis, L. (1996), Real Options, Managerial Flexibility and Strategy in Resource Allocation, MIT Press, Cambridge, Mass..

[18] Weisman, D. (1993), Superior Regulatory Regimes in Theory and in Practice, Journal of Regulatory Economics, 5, 355-66. 